\title{
Evaluación de síntomas en niños y adolescentes con cáncer: revisión integrativa
}

\author{
Evaluation of symptoms in children and teenagers with cancer: comprehensive review \\ Avaliação dos sintomas em crianças e adolescentes com câncer: uma revisão integrativa
}

\author{
Jennifer Sepúlveda-Ramírez* \\ Gloria Mabel Carrillo-González **
}

\section{Resumen}

Los niños y adolescentes diagnosticados con cáncer se enfrentan a una serie de cambios en los diferentes aspectos de su vida, y experimentan múltiples síntomas físicos y psicosociales a causa de la enfermedad y su tratamiento, lo que afecta su calidad de vida y la de sus cuidadores. Objetivo: Indagar el estado del aspecto investigativo relacionado con la temática sobre evaluación de síntomas en niños y adolescentes con cáncer. Método: Revisión integrativa de la producción científica generada entre 2007 y 2018 en las bases de datos Embase, Ovid, Medline, Pubmed, Science Direct y Scielo en inglés, español y portugués. Se incluyeron 50 artículos que aportan información sobre evaluación de síntomas en población pediátrica con diagnóstico de cáncer. Resultados: Las investigaciones sobre herramientas que evalúan la manifestación de síntomas, adaptadas y validadas a población pediátrica son limitadas. Conclusiones: Los niños y adolescentes con cáncer presentan síntomas durante las distintas fases de la enfermedad y el tratamiento. Son escasos los estudios sobre estos síntomas en el contexto local, por tanto se requiere validar escalas específicas para esta población, describir los síntomas incluyendo variables clínicas y de calidad de vida percibidas que sirvan para orientar intervenciones oportunas en el manejo de los mismos.

Palabras clave: Niño, neoplasia, síntomas cancerígenos, enfermería oncológica, evaluación de síntomas.

\footnotetext{
Abstract

Children and teenagers diagnosed with cancer have to face a series of changes in the different aspects of their life, and also experience multiple physical and psychosocial symptoms as a cause of the disease and its treatment, which affects their own life and their caregiver life quality. Objective: Investigate the current state of research related to the evaluation of symptoms in children and teenagers with cancer. Method: Comprehensive review about the scientific production in English, Spanish, and Portuguese languages generated between 2007 and 2018 in the databases Embase, Ovid, Medline, Pubmed, Science Direct and Scielo. 50 articles were included,
}

\section{Autor de correspondencia}

* $\triangle$ Enfermera. Magister en Enfermería. Universidad Nacional de Colombia. Sede Bogotá. Oncología Pediátrica. Correo: jsepulvedar@unal.edu. co. Orcid: https://orcid.org/0000-0001-6451-1297. Bogotá, Colombia.

** Doctora en Enfermería. Magister en Enfermería. Profesora Asociada Universidad Nacional de Colombia. Sede Bogotá. Facultad de Enfermería. Correo: gmcarrillog@unal.edu.co. Orcid: https://orcid. org/0000-0003-4513-104X. Bogotá, Colombia. 
ISSN-PRINT

1794-9831

E-ISSN 2322-7028

Vol. 16 No. 3

Sep - Dic 2019

Cúcuta, Colombia

which provide information about the evaluation of symptoms in the pediatric population diagnosed with cancer. Results: Researches about tools that evaluate the manifestation of symptoms, adapted and validated to pediatric population are limited. Conclusions: Children and teenagers with cancer show symptoms during different phases of the disease and treatment. The studies are scarce about the symptoms in the local context, therefore it is required to validate specific scales for this population, describe the symptoms including perceived clinical and life quality variables that can be used to orientate opportune interventions to handle the symptoms.

Key words: Child, neoplasm, cancer symptoms, oncology nursing, evaluation of symptoms.

\begin{abstract}
Resumo
As crianças e adolescentes diagnosticadas com câncer enfrentam-se a uma série de mudanças nos diferentes aspectos das suas vidas, experimentam sintomas físicos e psicossociais a causa da doença e o tratamento, afetando a sua qualidade de vida e a dos seus cuidadores. Objetivo: Indagar o estado das pesquisas relacionadas com a temática sobre a avalição dos sintomas de crianças e adolescentes com câncer. Métodos: Revisão integrativa da produção científica gerada entre 2007 e 2018 disponível em recursos bibliográficos na internet como Embase, Ovid, Medline, Pubmed, Science Direct e Scielo em inglês, espanhol e português. Incluíram-se 50 artigos que contribuíram com informação sobre avaliação de sintomas em população pediátrica com diagnostico de câncer Resultados: As investigações sobre as ferramentas que avaliaram manifestações dos sintomas, adaptadas e validadas na população pediátrica são limitadas. Conclusões: As crianças e adolescentes com câncer presentam sintomas durante as diferentes etapas da doença e o tratamento. São poucos os estudos sobre estes sintomas no contexto local, portanto, requerem-se instrumentos validados na população pediátrica, descrevendo os sintomas incluindo variáveis clínicas e de qualidade de vida percebidas que orientem intervenções oportunas para o acompanhamento dos mesmos.
\end{abstract}

Palavras-chave: Criança, neoplasia, sintomas, enfermagem oncológica, avaliação de sintomas.

\section{Introducción}

La tasa de incidencia de cáncer infantil en el mundo está entre 50-200 por cada millón de niños (1). De acuerdo a la Organización Panamericana de Salud (2): "la mayoría de los casos de cáncer infantil (65\%) se producen en América Latina y el Caribe donde se diagnostican 17.500 nuevos casos cada año y se registran más de 8.000 muertes a causa de esta enfermedad". En Colombia el cáncer infantil representa solo el $0.5 \%$ al $3 \%$ de los casos nuevos, sin embargo, tiene alta tasa de mortalidad, especialmente por leucemias agudas $(3,4)$.

A pesar de que el cáncer infantil muestra bajas tasas de incidencia, la mortalidad por esta enfermedad en menores de 15 años ha aumentado, pasando de un $2,2 \%$ de las defunciones en el periodo comprendido entre 1985-1989 a un 3,5\% en el periodo comprendido entre 2005-2008 (4). La mortalidad por cáncer infantil se ha relacionado con la presencia de diversos factores, tales como: la existencia de esfuerzos insuficientes para mejorar la atención integral que involucre el acceso a programas de detección temprana, diagnóstico oportuno y tratamiento pertinente disponible, especialmente en entornos de niños de bajos recursos; el abandono del tratamiento; y, la desinformación sobre la enfermedad, afectando de manera negativa la calidad de vida de los niños y sus familias (2).

En la actualidad, la efectividad de las terapéuticas ha contribuido a mejorar la supervivencia de los niños con cáncer, lo que ha generado la necesidad y el interés por desarrollar investigaciones que exploren a fondo las condiciones y factores a los que se exponen los pacientes durante el tratamiento indicado, como son los síntomas, el manejo de los efectos secundarios, el cambio de roles y la modificación en los estilos de vida en el hogar, entre otros aspectos $(5,6)$. Collins afirma que la comprensión de la prevalencia y características de los síntomas en niños con cáncer se ha visto obstaculizada por la falta de herramientas de evaluación validadas en esta población. La mayoría de los estudios se hacen mediante la medición de la calidad de vida para niños entre 12 a 18 años, sin que se específique la medición y evaluación de los síntomas.

De igual forma, para garantizar la atención integral del niño con cáncer y contribuir a mejorar las tasas de supervivencia, es necesario reconocer que ellos ex- 
perimentan múltiples síntomas físicos y psicológicos que independientemente de su etiología, comprometen y disminuyen su calidad de vida al punto de interferir con la continuidad del tratamiento (7-10).

Por otra parte, los niños y adolescentes diagnosticados con cáncer afrontan cambios en los diferentes aspectos de su vida, como lo son las actividades de la vida diaria, la escuela, la familia y su proceso de crecimiento y desarrollo; también, experimentan síntomas físicos y psicológicos a causa de la enfermedad y su tratamiento, lo que afecta su calidad de vida y la de sus cuidadores (11). Es por eso, que los síntomas se constituyen en una fuente de angustia para niños y adolescentes con cáncer y al aumentar la intensidad del tratamiento hace que se acreciente el riesgo de sufrir una alta carga de los mismos (12).

Para los adolescentes y niños con cáncer que reciben quimioterapia la exacerbación de los síntomas o efectos colaterales genera sufrimiento $(13,14)$. Los niños en algunas ocasiones manifiestan que comprenden los términos siendo incapaces de explicar los síntomas, lo que puede dificultar su atención (15).

Es importante indicar que la evaluación de los síntomas contribuye a mejorar las intervenciones para su control durante el tratamiento, en el marco de atención integral a los niños y adolescentes con cáncer. Por tanto, es importante reconocer esta necesidad ya que impactara positivamente en la vida de los sujetos de cuidado en oncología pediátrica (niños y familias).

Por otra parte, en el entorno hospitalario y ambulatorio se ha comprobado que existen dificultades para evaluar y controlar los síntomas debido a las interrupciones o cambios en la administración de medicamentos, en los cuidados, en el aislamiento de compañeros y de las actividades de la vida diaria, factores que hacen que la experiencia del síntoma sea negativa (16).

Hay que señalar que estos síntomas como entidades únicas rara vez ocurren solos, aparecen con frecuencia de manera simultánea y pueden interactuar entre sí, por lo que al identificar los grupos de síntomas en los escenarios de oncología pediátrica, puede proporcionar a las enfermeras mayor comprensión sobre la experiencia de los síntomas del paciente, permitiendo que se brinde apoyo y se planteen intervenciones eficaces $(5,10,13,17,18)$.
Cabe destacar, que acerca de los estudios sobre cáncer infantil, éstos se han centrado en investigar respecto a la supervivencia o toxicidad del tratamiento; sin embargo, existe la creciente necesidad de investigar sobre el impacto del tratamiento en los pacientes y su familia (7).

Es conveniente destacar, que la identificación de los grupos de síntomas ha sido más común en los estudios de oncología de adultos, mientras que la investigación sobre grupos de síntomas en niños y adolescentes es limitada. Esta situación ha generado la necesidad de investigar poniendo en evidencia un vacío del conocimiento con relación a la temática. El medir los síntomas en niños con cáncer infantil permitirá evaluar el funcionamiento físico y emocional durante la enfermedad y valorar cómo la presencia de los mismos interfiere con la calidad de vida que se debe brindar posteriormente a las intervenciones pertinentes, eficaces y oportunas.

\section{Metodología}

Revisión integrativa para determinar el estado actual del conocimiento sobre la evaluación de síntomas en niños y adolescentes con cáncer; se indagó en literatura empírica y teórica teniendo en cuenta los pasos descritos por Whittemore y Knafl (19).

1. Identificación del problema: Se delimitó el fenómeno de estudio relacionado con la evaluación de síntomas en niños y adolescentes con cáncer.

2. Búsqueda de literatura: Indagación bibliográfica generada entre 2007 y 2018 que permitió identificar 50 referencias relacionadas con la temática. Los términos de búsqueda fueron: síntoma, niño, cáncer, enfermería, síntomas cancerígenos, evaluación de síntoma. Las bases de datos utilizadas correspondieron a: Embase, Medline, Pubmed, Science Direct, Scielo, OVID Nursing, de donde se obtuvieron referencias en español, inglés y portugués. Se incluyeron artículos sobre investigaciones que tenían que ver con la experiencia del síntoma en niños con cáncer, en su mayoría a través de una herramienta para evaluar o monitorizar síntomas en dicha población; se excluyeron estudios realizados en población adulta.

3. Evaluación de datos: Se encontraron 70 referencias del fenómeno de evaluación de síntomas en niños con cáncer. Se excluyeron 20 artículos que relacionaban percepciones de padres o cuidadores sobre los sínto- 
ISSN-PRINT

1794-9831

E-ISSN 2322-7028

Vol. 16 No. 3

Sep - Dic 2019

Cúcuta, Colombia mas que presentaban los niños con cáncer y que planteaban guías para el manejo de síntomas. La evaluación de los datos se realizó a través de lectura crítica.

4. Análisis de datos: Se ordenaron, resumieron y consolidaron los datos relevantes en una matriz en la que se especificaron los conceptos, proposiciones, método y limitaciones de cada referencia.

5. Reducción de datos: Se organizaron referencias de acuerdo al tipo de diseño, características de la muestra y relevancia de las mismas.

6. Comparación de los datos: Se identificaron relaciones entre los datos de algunas referencias.

7. Conclusión y verificación: Se identificaron las similitudes y las diferencias, lo que permitió elaborar un conjunto de generalización de datos en relación al fenómeno de estudio. Se tuvo en cuenta la fiabilidad y validez de los estudios.

\section{Análisis}

Las publicaciones que exploran el área temática enfatizan en la pertinencia de estudiar los síntomas en la población pediátrica con cáncer, describiéndole como un fenómeno necesario para la investigación en oncología. Del total de las publicaciones se encontraron: 48 en inglés, 1 en español y 1 en portugués. En los artículos seleccionados predominan abordajes cuantitativos, diseños descriptivos y revisiones de literatura (Figura 1).

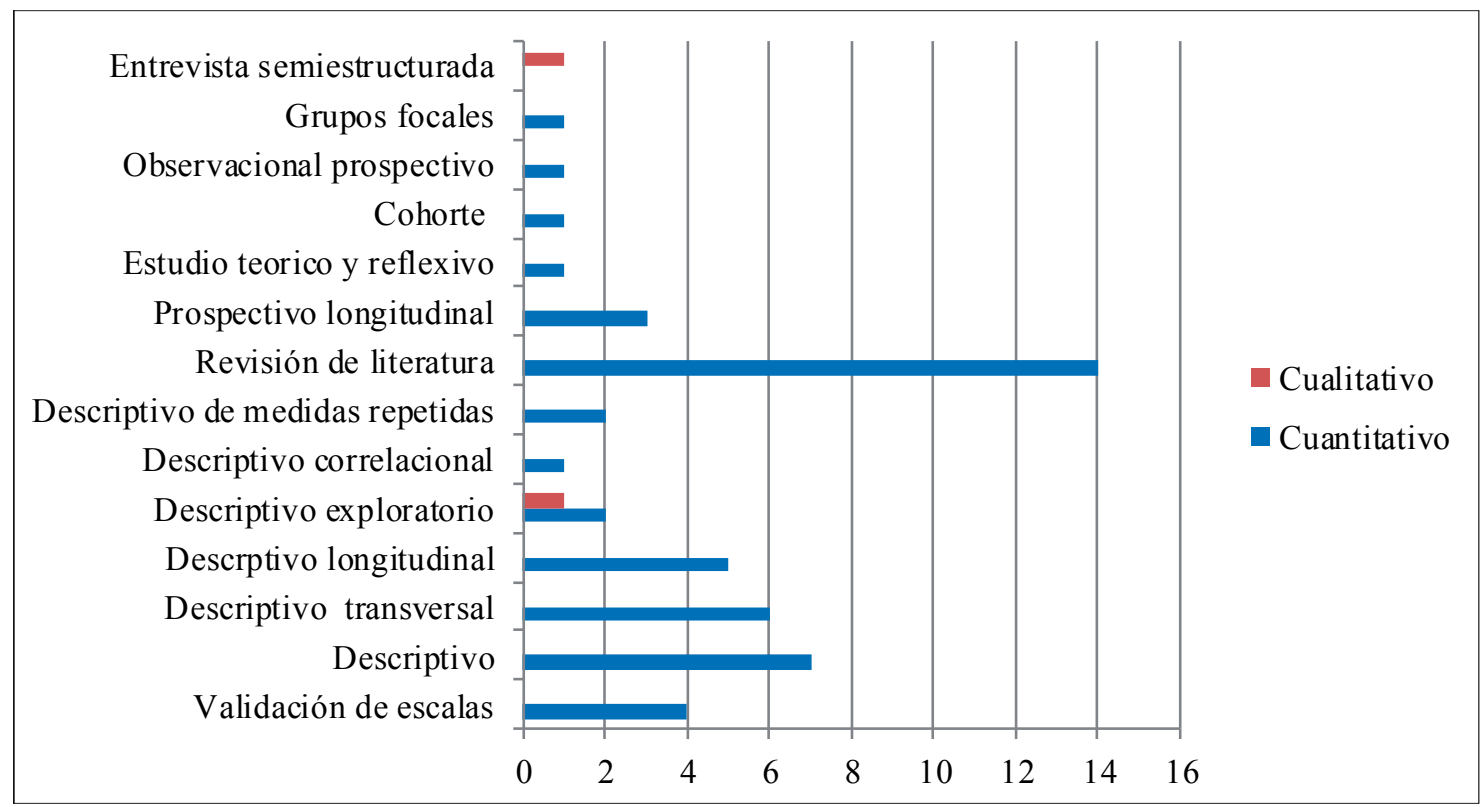

Figura 1. Diseños de estudios sobre evaluación de sintomas en niños y adolescentes con cáncer.

Fuente: Revisión de artículos sobre evaluación de síntomas en niños con cáncer. 2018.

\section{Instrumentos utilizados para medir síntomas en niños}

La comprensión sobre los síntomas y sus características en los niños con cáncer se ha visto obstaculizada debido a la falta de herramientas de evaluación adecuadas para esta población (17). Sin estos instrumentos, la epidemiologia del síntoma en cáncer infantil no puede estar caracterizada adecuadamente (7). Es por esto que existe la necesidad de identificar instru- mentos de autoinforme de síntomas con evidencia psicométrica, que logren comprender la experiencia del niño en relación al impacto del cáncer y el tratamiento en su vida (20).

Aunque algunos investigadores aplican dos o más instrumentos para evaluar estos síntomas, los datos pueden reflejar diferentes aspectos de la experiencia del síntoma, especialmente si se en gravedad, frecuencia o angustia (21). Algunos de estos instrumentos miden un síntoma específico como el dolor, las náuseas o la 
fatiga, tales como: Fatigue Scale-Adolescent (FS-A), Adolescent Pediatric Pain Tool, State-Trait Anxiety Inventory, Adolescent Pediatric Pain Tool (APPT) $(22-24)$.

Se puede señalar, que entre los instrumentos que se han aplicado para evaluar grupos o conjunto de síntomas se encuentran: Memorial Symptom Assessment Scale 7-12, Memorial Symptom Assessment Scale 10-18, Quality of Palliative Care QuestionnairePediatrics (Combinación de PedsQL y MSAS), SISOM, Therapy Related Symptom Checklist for Children (TRSC-C), The Center for Epidemiologic Studies Depression Scale for Children (CES-DC), Escala visual análoga (para valorar dolor, dificultad para dormir, náusea, apetito), Child Behavior Checklist/6-18 (CBCL), PedsQL y Proxy-report SSPedi, Patient-Reported Outcomes Measurement Information System (PROMIS) (Ver tabla 1).

Entre estos instrumentos la escala MSAS (Memorial Symptom Assessment Scale) ha sido una de las herramientas de mayor uso y ha demostrado ser válida $\mathrm{y}$ confiable en diversos contextos a escala mundial, en virtud de que permite evaluar la experiencia del síntoma en los niños y adolescentes con cáncer, contemplando la percepción y respuesta a dichos síntomas.

La escala MSAS fue desarrollada para proporcionar información sobre un grupo diverso de síntomas comunes en pacientes con cáncer, extraída de una revisión sobre literatura relacionada con dicha temática. Esta escala evalúa 32 síntomas físicos y psicológicos, dando origen a tres grupos de síntomas: psicológicos, físicos de alta prevalencia y físicos de baja prevalencia. Este instrumento es fiable y válido para evaluar la prevalencia, las características y el malestar de los síntomas en pacientes de oncología (25).

Por otra parte, la escala MSAS ha sido aplicada en: estudios relacionados con la calidad de vida en niños con cáncer (10), en niños que reciben tratamiento contra el cáncer $(5,25)$, en la identificación de grupos de síntomas en niños y adolescentes (25 - 27), en investigaciones con niños que padecen cáncer avanzado (16), para caracterizar la carga del síntoma en pacientes de oncología pediátrica (6) y ha sido validada en diferentes contextos a nivel mundial $(8,27,28)$.

Tabla 1. Investigaciones sobre instrumentos que miden síntomas en niños y adolescentes con cáncer.

\begin{tabular}{|c|c|c|c|c|}
\hline Autor/Año & Participantes & Instrumento/Escala & Síntoma & Resultado principal \\
\hline $\begin{array}{c}\text { Abu-Saad H, Sagherian } \\
\text { K, Tamim H. } \\
2013 \text { (9) }\end{array}$ & $\begin{array}{l}\text { Niños y adolescentes } \\
\text { entre } 7-18 \text { años }\end{array}$ & $\begin{array}{l}\text { - Quality of Palliative Care } \\
\text { Questionnaire-Pediatrics } \\
\text { (Combinación de PedsQL y } \\
\text { MSAS). }\end{array}$ & $\begin{array}{l}\text { Conjunto } \\
\text { de } \\
\text { síntomas }\end{array}$ & $\begin{array}{l}\text { Los puntajes más bajos en el PedsQL } \\
\text { fueron en náuseas y preocupación. } \\
\text { En los niños de } 7-12 \text { años, la falta } \\
\text { de apetito, el dolor y las náuseas } \\
\text { fueron predominantes. }\end{array}$ \\
\hline $\begin{array}{c}\text { Ameringer, S. } \\
\text { ElswickR.K } \\
\text { Shockey, DP, Dillon R. } \\
2013 \text { (29) }\end{array}$ & $\begin{array}{l}9 \text { adolescentes con } \\
\text { cáncer dentro de los } \\
6 \text { primeros meses del } \\
\text { diagnóstico. }\end{array}$ & $\begin{array}{l}\text { - Escala visual análoga (para valorar } \\
\text { dolor, dificultad para dormir, naúsea, } \\
\text { apetito). } \\
\text { - Fatigue Scale-Adolescent (FS-A). } \\
\text { - State-Trait Anxiety Inventory. }\end{array}$ & $\begin{array}{l}\text { Conjunto } \\
\text { de } \\
\text { síntomas }\end{array}$ & $\begin{array}{l}\text { Los principales síntomas a través de } \\
\text { los ciclos fueron fatiga, dificultad } \\
\text { para dormir y náuseas. La mayoría } \\
\text { de los adolescentes informaron } \\
\text { sobre ansiedad anticipada por recibir } \\
\text { quimioterapia. }\end{array}$ \\
\hline $\begin{array}{l}\text { Atay S, Conk Z, } \\
\text { Bahar Z. } \\
2012 \text { (30) }\end{array}$ & $\begin{array}{l}54 \text { niños recién } \\
\text { diagnosticados con } \\
\text { cáncer en Turquía. }\end{array}$ & $\begin{array}{l}\text { - Memorial Symptom Assessment } \\
\text { Scale 10-18. } \\
\text { - Cuestionario demográfico. }\end{array}$ & $\begin{array}{l}\text { Conjunto } \\
\text { de } \\
\text { síntomas. }\end{array}$ & $\begin{array}{l}\text { Los síntomas más comunes fueron: } \\
\text { falta de apetito, náuseas, letargo, } \\
\text { pérdida de cabello y sentimientos } \\
\text { de tristeza. Estos hallazgos pueden } \\
\text { usarse para desarrollar guías clínicas }\end{array}$ \\
\hline $\begin{array}{l}\text { Baggott C, Baird J, } \\
\text { Hinds P, Ruland CM, } \\
\text { Miaskowski C } \\
2015 \text { (31) }\end{array}$ & $\begin{array}{l}\text { Muestra a } \\
\text { conveniencia de niños } \\
\text { con cáncer entre } 7 \text { a } \\
12 \text { años. }\end{array}$ & $\begin{array}{l}\text { - SISOM } \\
\text { - Memorial Symptom Assessment } \\
\text { Scale. }\end{array}$ & $\begin{array}{l}\text { Conjunto } \\
\text { de } \\
\text { síntomas }\end{array}$ & $\begin{array}{l}\text { Los niños informaron un número } \\
\text { mayor de síntomas con Sisom en } \\
\text { comparación con el MSAS }\end{array}$ \\
\hline $\begin{array}{l}\text { Baggott C, Cooper BA, } \\
\text { Marina N, Matthay KK, } \\
\text { Miaskowski C. } \\
2012 \text { (17) }\end{array}$ & $\begin{array}{l}131 \text { niños y } \\
\text { adolescentes con } \\
\text { cáncer entre 10-18 } \\
\text { años de edad. }\end{array}$ & $\begin{array}{l}\text { - Memorial Symptom Assessment } \\
\text { Scale 10-18 }\end{array}$ & $\begin{array}{l}\text { Conjunto } \\
\text { de } \\
\text { síntomas }\end{array}$ & $\begin{array}{l}\text { Se identificaron tres grupos de } \\
\text { síntomas que se clasificaron como: } \\
\text { grupo de secuelas de quimioterapia, } \\
\text { grupo de trastornos del estado de } \\
\text { ánimo y malestar neuropsicológico. }\end{array}$ \\
\hline
\end{tabular}


ISSN-PRINT

1794-9831

E-ISSN 2322-7028

Vol. 16 No. 3

Sep - Dic 2019

Cúcuta, Colombia
William Li HC,

Williams PD, Lopez V,

Kwang Chung JO, Ying

Chiu S

2013 (27)

\begin{tabular}{|} 
Hedén L, Pöder, U, Von \\
Essen L, Ljungman G. \\
2013 (32) \\
\\
Linder LA, Al-Qaaydeh \\
S, Donaldson G. \\
2017 (12)
\end{tabular}

Williams PD, Williams AR, Kelly KP, Dobos C, Gieseking A, Connor R, Ridder L, Potter N, 2012 (33)

Williams PD, Robinson J, Williams AR 2014 (34)

Van Cleve L, Munoz

CE, Savedra M, Riggs

M, Bossert E, Grant M,

Adlard K

2012 (16)

Hyslop S, Dupuis L,

Baggott C, Dix D, Gibson P, 2018 (35)

Wang J, Jacobs D, Dewalt DA, Stem E, Gross H, Hinds PS. 2018 (36)

Reeye BB, Edwards LJ, Jaeger BC, Hinds PS, Dampier C, Gipson D, Selewski DT 2018 (37)
135 niños con cáncer entre 9-16 años en Hong-Kong.

160 padres de 89 niños con cáncer respondieron la escala en 6 momentos desde el diagnóstico del niño.

\section{0 niños y}

adolescentes que

recibían quimioterapia

en hospitalización.

385 niños y

adolescentes con leucemia linfoblástica aguda, tumores sólidos y tumores del SNC. entre 5-7 años con cáncer (LLA y otros).

\section{0 niños entre 6 a 17} años con diagnóstico

439 niños (8-18 años) y padres o tutores.

96 niños (8-18 años) con diagnóstico de cáncer
- Therapy Related Symptom Checklist for Children (TRSC-C)

- The Center for Epidemiologic Studies Depression Scale for Children (CES-DC)

- Memorial Symptom Assessment Scale (MSAS) 10-18 (version modificada)
- Memorial Symptom Assessment

79 niños hispánicos de cáncer avanzado. Scale 7-12.

Conjunto

de

síntomas

Conjunto

de

síntomas

Conjunto

de

síntomas

- Therapy Related Symptom Checklist for Children (TRSC-C)

- Therapy Related Symptom Checklist for Children (TRSC-C).

- The Common Toxicity Criteria Revised (CTC Revised)

- Memorial Symptom Assessment

Scale.

- Symptom Screening in Pediatrics Tool (SSPedi) for selfreport by children aged 8 to 18 years.

síntomas

Conjunto

de

síntomas

Conjunto

de

síntomas de energía son los síntomas más frecuentes. El dolor, la sensación de tristeza y las náuseas son los síntomas más angustiantes.

Surgieron dos dimensiones de experiencia de los síntomas, una fue síntomas individuales y dimensiones del síntoma a través del tiempo.

Los 5 síntomas más frecuentes: Conjunto sentirse lento, náuseas, pérdida de de apetito, irritabilidad y vómito. Los síntomas niños más grandes informaron una mayor gravedad de los síntomas.

La aparición y la gravedad de los síntomas en el TRSC-C en niños hispanos son consistentes con los hallazgos en estudios de otras etnias.

Los niños informaron dolor, náuseas, somnolencia y pérdida de energía en más del 50\% de las entrevistas.

SSPedi es confiable y válido en niños de 8 años a 18 años con cáncer.

Se identificaron dos perfiles: $52 \%$ síntomas menos graves y un $48 \%$ síntomas graves. Se evidencio una mejoría significativa de los síntomas después del tratamiento de quimioterapia.

Niños y adolescentes con cáncer entre 7-18 años con cáncer y síndrome nefrótico.
- Patient-Reported Outcomes Measurement Information System (PROMIS) de síntomas
- Patient-Reported Outcomes Measurement Information System (PROMIS)
Conjunto
de
síntomas

PROMIS tiene la capacidad para detectar cambios en la manifestación de los síntomas a lo largo del tiempo en diferentes momentos.

Fuente: Elaborada por autores.

\section{Prevalencia de los síntomas}

La prevalencia de los síntomas se ha explorado en niños $(14,17,35) \mathrm{y} / \mathrm{o}$ adolescentes $(10,14,17,38-40)$ que se encuentran en quimioterapia $(10,14,17,38,40)$, en cuidado paliativo (16), con cáncer avanzado, en población hispana (34), y particularmente con diagnóstico de leucemia linfoblástica aguda, tumores sólidos y tumores del Sistema Nervioso Central (SNC) $(10,41,42)$.
Es importante resaltar, que el personal de salud a menudo tiene dificultades para desarrollar estrategias sobre el manejo de los síntomas, más cuando la mayoría de las investigaciones sobre este tópico se centran en un solo síntoma como el dolor, la fatiga o la alteración del sueño $(23,24,42,43)$. En este sentido, se debe precisar que la fatiga es un síntoma con repercusión a nivel físico, cognitivo y psicológico (25). 
Del mismo modo, algunas de las investigaciones sobre cáncer infantil en relación a la atención de los síntomas se han efectuado especialmente en el contexto de los cuidados paliativos, siendo escasos los estudios sobre su atención en pacientes durante el tratamiento oncológico; sin embargo, es preciso mencionar que existen algunos que se enfocan en evaluar la forma de intervenir ante síntomas específicos como el dolor, la fatiga, mucositis, alteración del sueño y las náuseas principalmente (44 - 46). Los síntomas pueden manifestarse con mayor frecuencia o gravedad de acuerdo al tratamiento prescrito al niño.

Dentro de los síntomas físicos de mayor incidencia se reportan: náuseas, alteraciones del sueño, dolor, fatiga física, estreñimiento, diarrea, falta de apetito, pérdida de peso, somnolencia y boca seca $(5,14,17,44$, 47). En cuanto a los síntomas psicológicos de mayor ocurrencia se encuentran: fatiga mental, irritabilidad, nerviosismo, tristeza $(17,46)$. La fatiga en los niños (6-12 años) ha sido relacionada con una sensación física, mientras que en los adolescentes (13-18 años) se manifiesta en componentes mentales, emocionales y físicos (48). La evidencia demuestra que la presencia de estos síntomas se ha convertido en un factor estresante para la vida del niño con cáncer (49).

Finalmente, se debe subrayar que la comprensión sobre grupos de estos síntomas ha sido una temática ampliamente estudiada en adultos con cáncer; no obstante, ha ido surgiendo y tomando importancia su estudio en niños con la enfermedad. Por tal motivo, a medida que aumenta la sobrevida, es necesario promover la curación mientras se minimiza la toxicidad $(50,51)$. Se debe tener en cuenta que los grupos de síntomas que se manifiestan en niños y adolescentes con cáncer están relacionados con: la incomodidad sensorial e imagen corporal; alteraciones del sistema circulatorio y respiratorio relacionadas con la dificultad para respirar, fatiga, trastornos del sueño y depresión; grupo de secuelas de la quimioterapia (falta de energía, náuseas, vómito, cambios en la piel); y, el grupo de perturbación del estado de ánimo (11).

\section{Implicaciones para enfermería}

En la atención y cuidado del niño o adolescente con diagnóstico de cáncer se ha demostrado la importancia del rol de la enfermera en el proceso de identificación, evaluación y manejo de los síntomas a través de instrumentos adecuados y adaptados, durante el trata- miento y el curso de la enfermedad contribuyendo a garantizar el cuidado integral.

Sobre la temática, un grupo de enfermeras han desarrollado investigaciones que indagan sobre la experiencia de los síntomas y los grupos de síntomas; es decir, que se agrupan los síntomas que guardan alguna relación entre sí, lo que permite predecir la aparición de uno de ellos respecto a otro $(14,52)$. Estos estudios han permitido demostrar sobre la importancia de vincular a la práctica clínica intervenciones adaptadas a la condición del niño a partir de la comprensión de los síntomas.

En este sentido, se puede mencionar el desarrollo de investigaciones relacionadas con la evaluación de síntomas en niños con cáncer a través del uso de las tecnologías de la información y la comunicación (TIC), utilizando como herramienta el SISOM $(31,53)$. Dentro de estas investigaciones esta la efectuada por un grupo de enfermeras norteamericanas, que explora los síntomas a través de una multimedia animada para niños. Los hallazgos utilizando esta herramienta se contrastan con los de la escala MSAS que también es aplicada en niños.

De igual forma, se encuentra la herramienta Symptom Screening in Pediatrics Tool (SSPedi) construida y validada desde el año 2014 al 2017 y adaptada a niños entre 8-18 años que reciben terapia contra el cáncer $(34,54,55)$. Se aplica en escenarios como trasplante de células madre hematopoyéticas en donde los niños más grandes manifestaron mayor gravedad en los síntomas (56). También, está el instrumento C-SCAT (Computerized Symptom Capture Tool) desarrollado por enfermeras, con el fin de integrar tecnología innovadora y significativa para estudiar los síntomas y los grupos de síntomas en adolescentes con cáncer (57).

En definitiva, los planteamientos anteriores, evidencian dos aspectos relevantes: en primer lugar, el relacionado con la importancia que ha tomado el uso de estas herramientas elaboradas y disponibles en las diferentes plataformas relacionadas con las TIC, teniendo en cuenta que actualmente en los diferentes contextos padres y niños tienen acceso a las mismas; estas herramientas generalmente han sido adaptadas al contexto en que serán aplicadas (58). En segundo lugar, se ha convertido en una necesidad investigar sobre la aplicación de instrumentos adaptados y válidos que permitan identificar, monitorizar y evaluar los síntomas que presentan los niños y/o adolescentes durante su enfermedad y su terapia contra el cáncer e 
ISSN-PRINT

1794-9831

E-ISSN 2322-7028

Vol. 16 No. 3

Sep - Dic 2019

Cúcuta, Colombia incluso síntomas que experimentan siendo sobrevivientes de la enfermedad (59), en los diferentes escenarios de oncología pediátrica.

\section{Conclusiones}

- La revisión de literatura sobre investigaciones relacionadas con la evaluación de síntomas en niños y adolescentes con cáncer, permite demostrar que la aplicación de instrumentos es necesaria para generar intervenciones que mejoren la calidad de vida de los pacientes, teniendo en cuenta que la enfermedad y el tratamiento afectan significativamente su bienestar psicosocial y físico. La quimioterapia ha contribuido a mejorar las tasas de supervivencia, pero para que la terapia continúe con éxito, los efectos adversos del tratamiento requieren monitoreo, cuidados y manejo. La información basada en la evidencia que existe para la población pediátrica es limitada y sobre niños de habla hispana es aún más escasa $(30,34)$.

- El desarrollo de investigaciones centradas en temas relacionados con cáncer infantil es una necesidad como se ha señalado. Brindar atención integral al niño con cáncer y a su familia, tendrá repercusión en el aumento de tasas de supervivencia y curación, mayor adherencia al tratamiento y mejorará la experiencia de salud y calidad de vida de los niños y adolescentes con la enfermedad.

- Para los profesionales del área de la salud es determinante reconocer y manejar los síntomas, al tener un contacto directo y cercano con su sujeto de cuidado en los servicios de oncología pediátrica en el ámbito hospitalario y ambulatorio.

- La aplicación de instrumentos válidos para la evaluación de los síntomas puede representar también un indicador empírico para estudiar tanto la teoría como el modelo del manejo del síntoma (60) y así sustentar la práctica de enfermería en escenarios de oncología pediátrica.

Financiación: Universidad Nacional de Colombia. Proyecto desarrollado en el Marco de la Tesis de Maestría Adaptación cultural y validez de la "Memorial Symptom Assessment Scale (MSAS) 10-18" para Pediatría Oncológica en Colombia.

\section{Conflicto de intereses}

Las autoras declaran no tener ningún conflicto de interés.

\section{Referencias Bibliográficas}

1. Organización Mundial de Salud (OMS). El cáncer infantil. [Internet]. 2016 [consultado 25 de abril de 2017]. Disponible en: http://www.who.int/cancer/es/

2. Organización Panamericana de la Salud (OPS). El cáncer infantil en las Américas. [Internet]. 2014 [consultado 25 de abril de 2017]. Disponible en: https://www.paho.org/hq/dmdocuments/2014/OPSNota-Informativa-Cancer-Infantil-2014.pdf

3. Huertas Quintero J, Rivillas García J, Vera Rey A, Roldán Sánchez O, Ospina Martínez M. Guía metodológica para el observatorio Nacional de Cáncer [Internet]. 2015 [consultado 27 de Mayo de 2017]; Disponible en: https://www.minsalud.gov.co/sites/rid/Lists/BibliotecaDigital/RIDE/VS/ED/ GCFI/guia-ross-cancer.pdf

4. Piñeros M, Gamboa O, Suárez A. Mortalidad por cáncer infantil en Colombia durante 1985 a 2008. Revista Panamericana de Salud Pública. 2011; 30(1):15-21.

5. Hockenberry M, Hooke MC. Symptom clusters in children with cancer. Seminars in oncology nursing, 2007; 23(2):152-157.

6. Barz Leahy A, Feudtner C, Basch E. Symptom Monitoring in Pediatric Oncology Using Patient-Reported Outcomes: Why, How, and Where Next. Patient. 2018; 11(2):147-153.

7. Collins JJ, Byrnes ME, Dunkel IJ, Lapin J, Nadel T, et al. The measurement of symptoms in children with cancer. Journal of Pain Symptom Management. 2000; 19(5): 363-77.

8. Collins JJ , Devine TD, Dick GS, Johnson EA, Kilham HA, et al. The measurement of symptoms in 
young children with cancer: The validation of the memorial symptom assessment scale in children aged 7-12. Journal of Pain Symptom Management. 2002; 23(1): 10-6.

9. Abu Saad H, Sagherian K, Tamim H. Quality of life and symptom prevalence as reported by children with cancer in Lebanon. European Journal of Oncology Nursing. 2013; 17(6): 704-710.

10. Arslan FT, Basbakkal Z, Kantar M. Quality of life and chemotherapy-related symptoms of Turkish cancer children undergoing chemotherapy. Asian Pacific Journal of Cancer Prevention. 2013; 14(3): 1761-1768.

11. Rodgers C, Hooke MC, Ward J, Linder LA. Symptom Clusters in Children and Adolescents with Cancer. Seminars in Oncology Nursing. 2016; 32(4): 394-404.

12. Linder LA, Al Qaaydeh S, Donaldson G. Symptom Characteristics Among Hospitalized Children and Adolescents With Cancer. Cancer Nursing. 2017; 41(1): 23-32.

13. Erickson J, MacPherson CF, Ameringer S, Baggott C, Linder L, Stegenga K. Symptoms and symptom clusters in adolescents receiving cancer treatment: A review of the literature. International Journal of Nursing Studies. 2013; 50(6): 847-69

14. De Chico Cicogna E, Castanheira Nascimento L, García de Lima RA. Children and Adolescents with Cancer: Experiences with Chemotherapy. Revista Latino-Americana de Enfermagem. 2010; 8(5):86472.

15. Vatne TM, Slaugther L, Ruland CM. How Children with Cancer Communicate and Think About Symptoms. Journal of Pediatric Oncology Nursing. 010; 27(1) 24-32.

16. Van Cleve L, Munoz CE, Savedra M, Riggs M, Bossert E, Grant M, Adlard K. Symptoms in Children With Advanced Cancer. Child and Nurse reports. Journal of Pediatric Oncology Nursing. 2012; 29(1): 28-36.

17. Baggott C, Cooper BA, Marina N, Matthay KK, Miaskowski C. Symptom Cluster Analyses Based on Symptom Occurrence and Severity Ratings Among Pediatric Oncology Patients During Myelosuppressive Chemotherapy. Cancer Nursing. 2012; 35(1):19-28.

18. Williams PD, Piamjariyakul U, Shanberg R, Williams AR. Monitoring and Alleviation of Symptom Occurrence and Severity Among Thai Children and Adolescents During Cancer Treatments. Journal of Pediatric Oncology Nursing. 2015: 1-12.

19. Whittemore R, Knafl K. The integrative review: updated methodology. Journal of Advanced Nursing. 2005; 52(5):546-553

20. Pinheiro LC, McFatrich M, Lucas N, Walker JS, Withycombe JS, et al. Child and adolescent selfreport symptom measurement in pediatric oncology research: a systematic literature review. Quality of life research. 2018; 27(2): 291-319.

21. Baggott C, Dodd M, Kennedy C, Marina N, Miaskowski C. Multiple Symptoms in Pediatric Oncology Patients: A Systematic Review. Journal of Pediatric Oncology Nursing. 2009; 26(6): 325-339.

22. Fernandes A, Batalha L, Perdigao A, De Campos C, Nascimento L, Jacob E. Validação cultural do Adolescent Pediatric Pain Tool (APPT) em crianças portuguesas com cancro. Revista de Enfermagem Referência. 2015; 4(4): 99-105.

23. Dupuis LL, Ethier MC, Tomlinson D, Hesser T, Sung L. A systematic review of symptom assessment scales in children with cancer. BiomedCentral Cancer. 2012; 26(12): 430

24. Rodrigues Nunes MD, Miyauti Silva MC, Leonardo Rocha E, García de Lima RA, Castanheira Nascimento L. Measurement of fatigue in children and adolescents with cancer: an integrative review. Text \& Context Nursing Journal. 2014; 23(2): 492-501.

25. Portenoy RK, Thaler HT, Kornblith AB, McCarthy Lepore J, Friedlander Klar H, et al. The Memorial Symptom Assessment Scale: an instrument for the evaluation of symptom prevalence, characteristics and distress. European Journal of Cancer. 1994; 30(9):1326-1336. 
ISSN-PRINT

1794-9831

E-ISSN 2322-7028

Vol. 16 No. 3

Sep - Dic 2019

Cúcuta, Colombia
26. Yeh CH, Chiang YC, Chie LC, Lin L, Yang CP, Chuang HL. Symptom Clustering in Older Taiwanese Children with Cancer. Oncology Nursing Forum. 2008; 35(2): 273-281.

27. William Li HC, Williams PD, Lopez V, Kwang Chung JO, Ying Chiu S. Relationships among therapy-related symptoms, depressive symptoms, and quality of life in Chinese children hospitalized with cancer: an exploratory study. Cancer Nursing. 2013; 36(5):346-54.

28. Abu Saad H, Sagherian K, Tamim H. Validation of the Arabic Version of the Memorial Symptom Assessment Scale among Lebanese Cancer Patients. Journal of Pain Symptom Management. 2015; 50(4):559-65.

29. Ameringer S, Elswick RK, Shockey DP, Dillon R. A Pilot Exploration of Symptom Trajectories in Adolescents With Cancer During Chemotherapy. Cancer Nursing. 2013; 36 (1): 60-71.

30. Atay $S$, Conk $Z$, Bahar Z. Identifying symptom clusters in paediatric cancer patients using the Memorial Symptom Assessment Scale. European Journal of Cancer Care. 2012; 21(4):460-8.

31. Baggott C, Baird J, Hinds P, Ruland CM. Miaskowski C. Evaluation of Sisom: A computer-based animated tool to elicit symptoms and psychosocial concerns from children with cancer. European Jorunal of Oncology Nursing. 2015; 19(4): 359-369.

32. Hedén L, Pöder, U, Von Essen L, Ljungman G. Parents' Perceptions of Their Child's Symptom Burden During and After Cancer Treatment. Journal of Pain and Symptom management. 2013; 46(3): 366375.

33. Williams PD, Williams AR, Kelly KP, Dobos C, Gieseking A, et al. A symptom checklist for children with cancer. The Therapy-Related Symptom Checklist-Children. Cancer Nursing. 2012; 35(2): 89-98.

34. Williams PD, Robinson, J, Williams AR. Symptom occurrence and severity on the therapy-related symptom checklist for children among Hispanic pediatric oncology outpatients. Cancer Nursing. 2014; 37(3):12-20.

35. Hyslop S, Dupuis L, Baggott C, Dix D, Gibson P, et al. Validation of the Proxy Version of Symptom Screening in Pediatrics Tool in Children Receiving Cancer Treatments. 2018. 1-6.

36. Wang J, Jacobs D, Dewalt DA, Stem E, Gross H, Hinds PS. A Longitudinal Study of PROMIS Pediatric Symptom Clusters in Children Undergoing Chemotherapy. Journal of Pain and Symptom Management. 2018; 55(2): 329-367.

37. Reeye BB, Edwards LJ, Jaeger BC, Hinds PS, Dampier C, et al. Assessing responsiveness over time of the PROMIS pediatric symptom and function measures in cancer, nephrotic syndrome, and sickle cell disease. Quality of Life Research. 2018. 27: 249-257.

38. Hockenberry MJ, Hooke MC, Gregurich M, McCarthy K, Sambuco G, Krull K. Symptom Clusters in Children and Adolescents Receiving Cisplatin, Doxorubicin, or Ifosfamide. Oncology Nursing Forum. 2010; 37(1): 16-27.

39. Kim B, White K, Patterson P. Understanding the experiences of adolescents and young adults with cancer: A meta-synthesis. European Journal of Oncology Nursing. 2016; 24: 39-53.

40. Kestler SA, LoBiondo Wood G. Review of Symptom Experiences in Children and Adolescents With Cancer. Cancer Nursing. 2012; 35(2): 31-49.

41. Yeh $\mathrm{CH}$, Wang CH, Chiang YC, Lin L, Chien LC. Assessment of Symptoms Reported by 10- to 18-YearOld Cancer Patients in Taiwan. Journal of Pain and Symptom Management. 2009; 38(5):738-746.

42. Linder LA, AmeringerS, Baggott C, Erickson J, Machpherson C, et al. Measures and methods for symptom and symptom cluster assessment in Adolescents and Young Adults with Cancer. Seminars in Oncology Nursing. 2015; 31(3): 206-215.

43. Miyauti da Silva MC, Lopes Júnior LC, Castanheira Nascimento L, García de Lima RA. Fatiga en niños y adolescentes con cáncer bajo la perspectiva de los profesionales de salud. Revista LatinoAmericana Enfermagem. 2016; 24: 1-10. 
44. Tomlinson D, Hinds PS, Ethier MC, Ness KK, Zupanec S, Sung L. Psychometric Properties of Instruments Used to Measure Fatigue in Children and Adolescents With Cancer: A Systematic Review. Journal of Pain Symptom Management. 2013; 45(1): 83-91.

45. McCulloch R, Hemsley J, Kelly P. Symptom management during chemotherapy. Paediatrics and child health. 2018; 28(4): 189-195.

46. Wolfe J, Orellana L, Ullrich C, Cook F, Kang TI, et al. Symptoms and Distress in Children with Advanced Cancer: Prospective Patient-Reported Outcomes From the PediQUEST Study. Journal of Clinical Oncology. 2015; 33 (17): 1928-1935.

47. Miller E, Jacob E, Hockenberry MJ. Nausea, Pain, Fatigue, and Multiple Symptoms in Hospitalized Children with Cancer. Oncology Nursing Forum. 2011; 38(5): 82-93

48. Hooke MC, Garwick AW, Gross CR. Fatigue and Physical Performance in Children and Adolescents Receiving Chemotherapy. Oncology Nursing Forum. 2011; 38 (6): 649-657.

49. Hockenberry M. Symptom Research in Children with Cancer: One Researcher's Journey. Virginia Henderson Global Nursing Repository. 2017.

50. Hockenberry MJ, Hooke MC, Rodgers C, Taylor O, Koerner KM, et al. Symptom Trajectories in Children Receiving Treatment for Leukemia: A Latent Class Growth Analysis with Multitrajectory Modeling. Journal of Pain and Symptom Management. 2017; 54(1): 1-8.

51. Miaskowski, C. Future directions in symptom cluster research. Seminars in Oncology Nursing. 2016; 32(4): 405-415.

52. Lopes Júnior LC, De Omena Bomfim E, Castanheira Nascimento L, Pereira da Silva G, García de Lima RA. Theory of unpleasant symptoms: support for the management of symptoms in children and adolescents with cancer. Revista Gaúcha de Enfermagem. 2015; 36(3):109-112.

53. Tsimicalis A, Rennick J, Stinson J, Le May S, Louli J, et al. Usability testing of an interactive communication tool to help children express their cancer symptoms. Journal of Pediatric Oncology Nursing. 2018: 1-12.

54. Tomlinson L, Dupuis LL, Gibson D, Johnston DL, Portwine C, et al. Initial development of the Symptom Screening in Pediatrics Tool (SSPedi). Support Care Cancer. 2014; 22: 71-75.

55. O'Sullivan L, Dupuis L, Gibson P, Johnston DL, Baggott C, et al. Refinement of the Symptom Screening in Pediatrics Tool (SSPedi). British Journal of Cancer. 2014; 111: 1262-1268.

56. Johnston DL, Hyslop, S, Tomlinson D, Baggott C, Gibson $\mathrm{P}$, et al. Describing symptoms using the Symptom Screening in Pediatrics Tool in hospitalized children with cancer and hematopoietic stem cell transplant recipients. Cancer Medicine. 2018; 7(5):1750-1755.

57. Linder L, Macpherson CF, Stegenga K, Ameringer S, Erickson J, et al. Feasibility and acceptability of an iPad application to explore symptom clusters in Adolescents with cáncer. Virginia Henderson Global Nursing e-Repository. 2018.

58. Wang J, Yao N, Liu Y, Geng Z, Wang Y, et al. Development of a Smartphone Application to Monitor Pediatric Patient-Reported Outcomes. Computers, Informatics, Nursing. 2017; 35 (11): 590-598.

59. Menard JC, Hinds PS, Jacobs SS, Cranston K, Wang J, et al. Feasibility and Acceptability of the Patient-Reported Outcomes Measurement Information System Measures in Children and Adolescents in Active Cancer Treatment and Survivorship. Cancer Nursing. 2014; 37(1): 66-74.

60. Humphreys J, Janson S, Donesky D, Dracup K, Lee K, et al. A Middle Range Theory of Symptom Management. En: Smith MJ, Liehr PR. Middle Range Theory for Nursing. Third edition. New York: Springer Publishing Company. 2014. 